\title{
Data Analysis and reduction using stationary solutions of the NLS equation
}

\author{
Bernard Deconinck* and David O. Lovit ${ }^{\dagger}$ \\ Department of Applied Mathematics, University of Washington, Seattle WA 98195-2420
}

(December 10, 2009)

\begin{abstract}
We demonstrate that the stationary solutions of the Nonlinear Schrödinger Equation (NLS) give rise to families of orthonormal bases for the square integrable functions with periodic boundary conditions. This allows us to use these solutions in much the same way as regular Fourier modes or other basis sets. For instance, we show the applicability of the NLS basis sets for doing data analysis, and as a means for obtaining reduced models. Using a variety of examples, we show that working with the NLS basis sets is advantageous over working with the set of Fourier modes, which they contain as a special case. The NLS sets are particularly appealing for those problems where the NLS equation is expected to provide a good description of any underlying dynamics, such as the description of modulated waves in deep water or nonlinear optics. In those cases, the decomposition of experimental data requires fewer modes compared to regular Fourier analysis, and the truncated components remain unimportant on longer time scales.
\end{abstract}

Keywords: Nonlinear Schrödinger equation, complete basis, data analysis, modal reduction

\section{Introduction}

There is no need to convince the reader that the explicit knowledge of a complete orthonormal basis for an infinite-dimensional function space facilitates many different processes in that space. The classical example is that of the set of Fourier modes in the space of square-integrable functions on a finite interval, with suitable boundary conditions. Having such a basis we may consider doing different things. Two examples are:

- We can perform the spectral analysis of any function in the space in terms of the basis functions or modes. The nature of the function being analyzed determines how effective the given basis is for its spectral (or data) analysis [5, 14]: how fast does the truncation error approach zero as more modes are taken into account?

- If any dynamics is prescribed on the function space, we may do a modal reduction of the infinite-dimensional dynamics by limiting ourselves to the dynamics of a low number of modes. The effectiveness of this approach is determined both by how many modes are required to accurately describe initial conditions, and by how long the finite-dimensional dynamics approximates the infinite-dimensional dynamics. A famous example of such a modal reduction is given by the Lorenz system [19], where Fourier modes were used to obtain a three-dimensional description.

\footnotetext{
*Email: bernard@amath.washington.edu

†Email: davidlovit@gmail.com
} 
In this paper, we present new families of complete orthonormal bases for the periodic square integrable functions. The elements of each basis are stationary solutions of the Nonlinear Schrödinger equation (NLS)

$$
i u_{t}=-u_{x x}-2 \sigma|u|^{2} u,
$$

where $u$ is a complex-valued function, and $\sigma$ is 1 (focusing NLS) or -1 (defocusing NLS). We refer to the new bases as NLS bases. Although any function can be decomposed as a linear combination of the elements of any of the NLS bases, the unexpected connection between a linear concept (a basis) and a nonlinear one (stationary solutions of a nonlinear partial differential equation) hints at the usefulness of the new bases. Specifically, for those applications where the NLS equation or a perturbation of it is expected to be a good model, we anticipate the use of the NLS bases to be beneficial in that few modes may give an accurate description, both in terms of data analysis and in terms of modal reduction. Different applications are examined in this paper.

As is shown below, the different NLS bases are characterized by a parameter $k$, the elliptic modulus of the NLS stationary solutions. For each $k \in[0,1)$ a different basis is obtained. Choosing $k=0$ results in the Fourier basis, thus it is clear that the Fourier basis is obtained as a specific instance of the NLS bases. This implies that applications such as data analysis using the NLS bases will never yield results worse than are obtained using Fourier modes. In practice, the choice of NLS basis is determined by minimizing an error functional over the parameter $k$.

\section{Stationary Solutions of NLS}

In this section we derive the stationary solutions of the Nonlinear Schrödinger Equation. The results are not new. They are presented in $[2,3,6]$, for example. Nevertheless, it is beneficial to include them here for what follows. Stationary solutions of NLS (1) are of the form

$$
u=e^{-i \omega t+i \theta(x)} r(x) .
$$

Substitution of this ansatz in (1), followed by dividing by the omnipresent exponential factor, the separation of the real and imaginary parts yields

$$
\begin{aligned}
0 & =\theta^{\prime \prime} r+2 \theta^{\prime} r^{\prime}, \\
\omega r & ={\theta^{\prime}}^{2} r-r^{\prime \prime}-2 \sigma r^{3} .
\end{aligned}
$$

We rewrite $(3)$ as $\left(\ln \theta^{\prime}\right)^{\prime}=-2(\ln r)^{\prime}$ to find that $\theta^{\prime}=c r^{-2}$, where $c$ is an integration constant. Then (4) becomes

$$
\omega r=c^{2} r^{-3}-r^{\prime \prime}-2 \sigma r^{3}
$$

We multiply by $r^{\prime}$ and integrate to obtain 


$$
\frac{1}{2} \omega r^{2}=-\frac{1}{2} c^{2} r^{-2}-\frac{1}{2} r^{\prime 2}-\frac{1}{2} \sigma r^{4}+\frac{d}{8}
$$

where $d$ is an arbitrary constant. Setting $s=r^{2}$, we have

$$
s^{\prime 2}=-4 c^{2}-4 \omega s^{2}-4 \sigma s^{3}+d s .
$$

This equation for $r$ is solved using elliptic functions of the form

$$
r^{2}=A \operatorname{sn}^{2}(x, k)+B
$$

where $\operatorname{sn}(x, k)$ is the Jacobi elliptic sine function with elliptic modulus $k \in[0,1)$ $[16,25]$. Finally, we obtain the explicit solution

$$
u(x, t)=\sqrt{-\frac{k^{2}}{\sigma} \operatorname{sn}^{2}(x, k)+B} \cdot \exp \left(i c \int_{0}^{x} \frac{d z}{-\frac{k^{2}}{\sigma} \operatorname{sn}^{2}(z, k)+B}-i \omega t\right),
$$

where

$$
\begin{gathered}
c^{2}=\sigma B(\sigma B-1)\left(\sigma B-k^{2}\right), \\
\omega=1+k^{2}-3 \sigma B
\end{gathered}
$$

and $B$ is a free parameter. Note that $|u(x, t)|$ is periodic in $x$ with period $2 K(k)$, where $K(k)$ is the complete elliptic integral of the first kind $[16,25]$ :

$$
K(k)=\int_{0}^{\pi / 2} \frac{1}{\sqrt{1-k^{2} \sin ^{2} z}} d z
$$

A special case of these stationary solutions is presented by the so-called trivialphase $^{1}$ solutions,

$$
\begin{array}{lrl}
u(x, t)=k \operatorname{cn}(x, k) e^{i\left(2 k^{2}-1\right) t}, & \text { for } \sigma=1, \\
u(x, t)=\operatorname{dn}(x, k) e^{i\left(2-k^{2}\right) t}, & \text { for } \sigma=1, \\
u(x, t)=k \operatorname{sn}(x, k) \exp \left(-i\left(1+k^{2}\right) t\right), & \text { for } \sigma=-1 .
\end{array}
$$

In the literature, these solutions have traditionally received more attention than the nontrivial phase ones, because they are easier to investigate, due to their simplified

${ }^{1}$ Since these solutions correspond to values of $B$ for which $c=0$. 
functional form. For our purposes, we need all stationary solutions of the NLS equations, including the nontrivial phase ones.

Remark. The solution (5) is not the most general form of the stationary solution of the NLS equation. The equation has a number of classical Lie symmetries [21], which may be used to get this most general form. For instance, the solution may be multiplied by an arbitrary constant phase factor, or, more relevant for our considerations below, the variables and the solution may be multiplied by appropriate scaling constants, due to the scaling invariance of the NLS equation. Specifically, by letting $x \rightarrow \kappa x$, the modulus of the scaled solution becomes periodic with period $L=2 K(k) / \kappa$. Thus, for any positive choice of the period $L$ and for any given $k \in[0,1)$, it is easy to choose a scaling factor $\kappa$ to construct a stationary solution of the NLS solution that is periodic with period $L$ and is parameterized by the given elliptic modulus $k$.

\section{The Spectral Stability Problem for the Trivial-Phase Solutions and a basis for the square integrable periodic functions}

The stability of the solutions constructed above has been examined extensively, see for instance $[4,9,10]$. In this manuscript we are not interested in the stability of these solutions per se. Rather, we use the stability problem for the trivial-phase solutions to show that the stationary solutions of the NLS equation give rise to different bases for the square-integrable periodic functions of prescribed period $L$. Examining the stability of these nontrivial-phase solutions, we are led to an operator that proves useful to this end.

Consider a perturbation of a trivial-phase solution $\phi(x) e^{i \omega t}$ of the form

$$
u=e^{i \omega t}\left(\phi(x)+\epsilon v(x, t)+i \epsilon w(x, t)+\mathcal{O}\left(\epsilon^{2}\right)\right) .
$$

We do not impose restrictions on the class of perturbations other than that they are continuous and bounded on the whole line:

$$
u, v \in C_{b}^{0}(\mathbb{R})
$$

Substituting (11) into NLS, dropping the contributions that are at least quadratic in $\epsilon$ and taking real and imaginary parts, we obtain

$$
\begin{aligned}
& \omega w-2 \sigma \phi^{2} w-w_{x x}=v_{t}, \\
& -\omega v+6 \sigma \phi^{2} v+v_{x x}=w_{t} .
\end{aligned}
$$

Since the above system does not depend explicitly on $t$, we let

$$
\begin{aligned}
v(x, t) & =V(x) e^{\Omega t}+c . c ., \\
w(x, t) & =W(x) e^{\Omega t}+c . c .
\end{aligned}
$$

where c.c. denotes complex conjugate. The resulting system is written as 

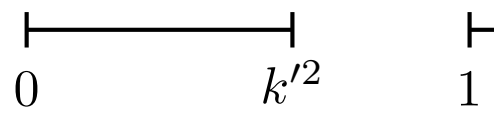

Figure 1. The essential spectrum of the one-gap Lamé operator (15).

$$
\left(\begin{array}{cc}
0 & L_{-} \\
-L_{+} & 0
\end{array}\right)\left(\begin{array}{c}
V \\
W
\end{array}\right)=\Omega\left(\begin{array}{c}
V \\
W
\end{array}\right)
$$

where

$$
L_{-}=-\partial_{x}^{2}+\omega-2 \sigma \phi^{2}
$$

and

$$
L_{+}=-\partial_{x}^{2}+\omega+6 \sigma \phi^{2} .
$$

These operators are considered on $C_{b}^{0}$, as remarked above. To be more concrete, let us suppose that $\sigma=-1$ (defocusing case) and we consider the stability of the solution (10). Then

$$
L_{-}=-\partial_{x}^{2}-1-k^{2}+2 k^{2} \operatorname{sn}^{2}(x, k) .
$$

In the focusing case, $L_{-}$is simply a constant shift of the $L_{-}$above, and thus has the same eigenfunctions. These eigenfunctions are what we are particularly interested in here, so we proceed to work with the operator

$$
L_{-}=-\partial_{x}^{2}+k^{2}\left(2 \operatorname{sn}^{2}(x, k)-1\right) .
$$

This is a one-gap Lamé operator, which is well studied [11]. Its essential spectrum is

$$
\sigma\left(L_{-}\right)=\left[0, k^{\prime 2}\right] \cup[1, \infty)
$$

as shown in Figure 1. Here $k^{\prime}$ is the complimentary elliptic modulus, $k^{\prime 2}=1-k^{2}$.

It can be verified by direct substitution that (5) gives two (because of the freedom in the sign of $c$ ) eigenfunctions of $L_{-}$with eigenvalue $\lambda$, provided we choose $B=(1-\lambda) \sigma$. In this case $c= \pm \sqrt{\lambda(\lambda-1)\left(\lambda-1+k^{2}\right)}$. If $\lambda=0, k^{2}=1-k^{2}$, or 1 , the eigenfunction (5) is a trivial-phase solution. For these eigenvalues only a single eigenfunction is obtained ${ }^{1}$, as $c=0$ and the choice of its sign is irrelevant. In short, direct substitution verifies that the stationary solutions of NLS are eigenfunctions of the linear operator $L_{-}$. What can we say about the properties of these eigenfunctions?

\footnotetext{
${ }^{1}$ A second solution of the differential problem may be obtained through reduction of order, but it is linearly growing.
} 
If we restrict ourselves to considering periodic eigenfunctions of period $L$, the spectrum reduces to a point subset of the essential spectrum shown in Figure 1, as is well known [20]. In this case, since the operator $L_{-}$is of Sturm-Liouville type, many properties of the eigenfunctions are known [17]:

- The eigenfunctions are mutually independent,

- The eigenfunctions are mutually orthogonal, and

- The eigenfunctions are complete in $L^{2}(0, L)$.

Thus we may use the stationary solutions of period $L$ (obtained using the scaling symmetry, as noted above) of the NLS equation as a basis for $L^{2}(0, L)$. We have constructed a one-parameter family of such bases, parameterized by the elliptic modulus $k$. It should be noted that this basis contains the Fourier basis as a special case. Indeed, if we equate the elliptic modulus $k$ to zero, the eigenfunctions (5) reduce to Fourier modes.

Remark. Relating to the modern point of view of Sturm-Liouville problems (see the introduction of [15]), we show that the function $u(x, t)$ is closely related to the Baker-Akhiezer function for $L_{-}$. First, we rewrite (5) with $B=(1-\lambda) \sigma$, using the identity $k^{2} \operatorname{sn}^{2}(x, k)+\operatorname{dn}^{2}(x, k)=1$, as

$$
u(x, t)=\sqrt{\operatorname{dn}^{2}(x, k)-\lambda} \cdot \exp \left(i c \int \frac{d x}{\operatorname{dn}^{2}(x, k)-\lambda}\right) .
$$

If we define $R_{0}(x, \lambda)=\sqrt{\left(\lambda-\operatorname{dn}^{2}(x, k)\right) /\left(\lambda-k^{\prime 2}\right)}$, then

$$
\psi(\lambda)=\frac{u}{\sqrt{k^{\prime 2}-\lambda}}=R_{0}(x, \lambda) \exp \left(i \sqrt{\frac{\lambda(\lambda-1)}{\lambda-k^{\prime 2}}} \int \frac{d x}{R_{0}^{2}(x, \lambda)}\right) .
$$

We show that $\psi(\lambda)$ is the Baker-Akhiezer function for $L_{-}$with parameter $\lambda^{-1 / 2}$ and divisor consisting of a single point at $1 / k^{\prime}$.

- First, we see that $\psi$ is an eigenfunction of $L_{-}$, since it is a multiple of $u$.

- Next, we consider the asymptotic behavior of $\psi(\lambda)$ as $\lambda \rightarrow \infty$. We see that $R_{0}(x, \lambda) \rightarrow 1$, and $\psi \rightarrow e^{i \sqrt{\lambda} x}$.

- Finally, $\psi$ has a pole at $\lambda=k^{\prime 2}$.

Note that, in addition, it can be seen that $\psi$ has one zero in the gap of the essential spectrum of $L_{-}$, at $\lambda=\operatorname{dn}^{2}(x, k)$, since $k^{\prime} \leq \operatorname{dn}(x, k) \leq 1$.

\section{Numerical and experimental applications}

We have established that the NLS stationary solutions of period $L$ give rise to a one-parameter family of bases for $L^{2}(0, L)$. Thus, we can write any function in $L^{2}(0, L)$ as a linear superposition of stationary solutions of the NLS equation. This is illustrated in Figure 2, where we see how the error in approximating sample data with eigenfunctions decays as the number of modes increases.

In the remainder of this paper we use this simple observation to use the NLS bases as a data analysis tool, and as a way of doing modal reduction. 


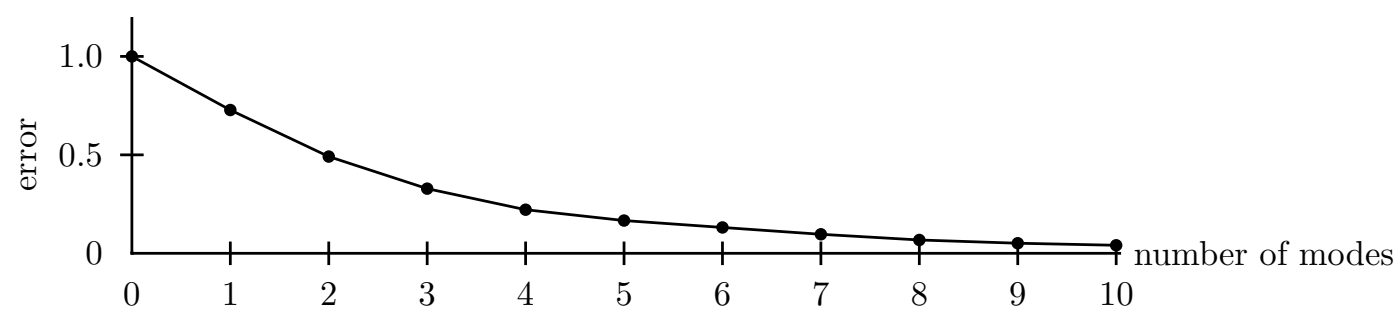

Figure 2. The typical decay of error (as a fraction of the 2-norm) of the eigenfunction approximation as the number of eigenfunctions used for the approximation increases. It can be shown that this decay is exponential.

\subsection{Numerical calculation of the eigenfunctions and data analysis}

In order to use the NLS bases for data analysis, it is necessary to have an efficient method to compute the basis functions, and the expansion coefficients of the data. The basis functions themselves involve Jacobi elliptic functions, and an integral of these. The expansion coefficients involve these functions in the integrand. The direct method of calculating these coefficients involves the numerical evaluation of a nested integral of elliptic functions and is computationally expensive, especially for high elliptic modulus.

Since the elements of the NLS bases are eigenfunctions of the linear operator $L_{-}$ which has periodic coefficients, we use the Floquet-Fourier-Hill method [7] for their computation. By Floquet's theorem, if we choose an eigenfunction $\phi$ with period $L$, we may write $\phi(x)=e^{i \mu x} \psi(x)$, where $\psi(x+L)=\psi(x)$, with $\mu \in[0,2 \pi / L]$. This method yields an approximation for each eigenfunction $\phi(x)$ as a truncated Fourier series

$$
\phi(x) \approx \sum_{j=-N}^{N} c_{j} e^{i x(\mu+2 \pi j / L)} .
$$

Projecting data becomes a simple and computationally efficient task: we take an FFT to find a truncated Fourier series for the data, and then take a dot product of the coefficients to project them onto the NLS basis corresponding to the value of $k$ we wish to use. In all computations that follow, this is the numerical method used for the eigenfunction expansions.

A few remarks are in order about how the NLS bases compare with the Fourier basis.

- As previously mentioned, the Fourier basis is a special case of the NLS bases. It is obtained from them by equating $k=0$. This implies that expansions of a given function in terms of the elements of either the Fourier basis or the different NLS bases always require at most as many terms using the appropriate NLS basis, compared to the Fourier basis, for a prescribed truncation error. Choosing $k=0$ results in the same number of terms. In almost all cases a different choice of $k$ allows for a better approximation with the same number of terms, or an equally good approximation with fewer terms. As noted before, varying the elliptic modulus changes the period of the elements of the NLS basis. This is compensated for by scaling the independent variable $x$.

- Instead of using the elliptic modulus $k \in[0,1)$, it is often useful to parameterize the elliptic functions using the nome $q \in[0,1)[25]$ : 


$$
q=e^{-\pi K\left(k^{\prime}\right) / K(k)} .
$$

The disadvantage of using the elliptic modulus $k$ is that it offers a poor parameterization of change in behavior of the elliptic functions as $k$ changes: the elliptic functions are essentially trigonometric functions for $k \in[0,0.95]$, and any change in their behavior occurs for $k>0.95$. Using the elliptic nome instead, this change in behavior is uniformized over $q \in[0,1)$. Indeed, note that $q=0.5$ corresponds to $k=0.99999476105 \ldots$

- The elliptic modulus $k$ (or the elliptic nome $q$ ) is not the only parameter describing the NLS bases. As remarked before, using the Lie symmetries, other parameters may be introduced. For instance, we may shift the independent variable, to center the basis functions at different locations. Note that for the Fourier basis a shift in the independent variable results in a different, but equivalent basis as it merely multiplies the Fourier coefficient by a unimodular constant. This is not true for the NLS bases.

\subsection{Data analysis: examples}

Although any square integrable function may be decomposed using different NLS bases, we expect the use of these bases to be especially beneficial in those situations where we encounter data from application settings where a perturbation of the NLS equation is supposed to be a good model. This is illustrated in this section.

\section{Remarks.}

- In most applications ${ }^{1}$ where the NLS arises it describes the dynamics of the envelope of a modulated wave train. For instance, this is true in deep water [24] and in nonlinear optics [12]. To this end, when we use the NLS bases for data analysis in such applications, we instead analyze the data by filtering out the fast carrier wave frequency of the modulated wave train. We refer to such data as demodulated data.

- The data analysis using solutions of NLS should not be confused with that found in [22]. There, signals are analyzed using so-called higher-genus solutions of the NLS equation. Our approach is far simpler: instead of matching a signal with a single higher-genus solution [22], we decompose a signal as a linear superposition of stationary solutions of the NLS equation. We know this process is convergent, due to the completeness of our bases.

Example 1. Deep-water wave-tank data. The data we consider here were kindly provided by Megan Catalano and Diane Henderson. The experiments were performed by them in the William G. Pritchard Fluid Mechanics Laboratory in the Mathematics Department at Penn State University. We restrict ourselves to comparisons using 4 Fourier modes and 4 NLS modes, to have distinguishable errors to consider. The improvements in error reduction are consistently maintained when more modes are used.

In Figure 3a, we compare demodulated water wave tank data from an experiment in the one-dimensional wave tank (i.e., a long narrow wave tank; see [24] for a description of the experimental set-up) in the laboratory with its projection onto four Fourier modes and onto four eigenfunctions. The elliptic nome $q$ is chosen by

\footnotetext{
${ }^{1}$ Bose-Einstein condensates are a notable exception
} 
a straightforward numerical optimization minimizing the error in the 2-norm. The reduction in error obtained from using a 4-dimensional NLS basis as opposed to a 4-dimensional Fourier basis in the first example (Figure 3a) is less than $30 \%$. This is not surprising: the elliptic nome is $q=0.242$, and the elliptic functions themselves are approximated relatively well using 4 Fourier modes.

A better result might be expected for the experiment of Figures 3b-c. These experiments were performed in the two-dimensional (i.e., the wave surface has genuine dependence on both horizontal spatial variables) wave basin in the Pritchard Laboratory. A description of the experimental set-up can be found in [8]. For the experiment of Figure $3 \mathrm{~b}$ the elliptic nome is $q=0.928$. In this case, the reduction in error is $43 \%$. For the last experiment (Figure 3c) the elliptic nome is $q=0.454$. On the other hand, the data of this experiment displays the steepest wave profile, thus nonlinear effects are expected to matter. This is confirmed by a reduction in error of well over $70 \%$. These experiments demonstrate that the NLS basis with the right elliptic nome outperforms the Fourier basis as it should, with significant decreases in the measured error when using an equal number of modes.

Example 2. Typhoon Krosa (2007) data. Our second example is not from a controlled experiment. Instead we use demodulated field data, kindly provided to us by Paul C. Liu (NOAA), who obtained them from the Central Weather Bureau (CWB) of Taiwan. The original data are obtained from buoy measurements recorded at one hour intervals at the height of Typhoon Krosa, 2007. The two time series used here correspond to wave trains separated by three hours. The first time series (Figure 4) contains one of the largest wave heights ever recorded [18], with a trough to crest wave height of 32.3 meters. The second time series (Figure 5) displays the presence a wave packet with amplitude exceeding nearby packet amplitudes by several factors. Both time series were measured for 512 seconds. It is clear to the eye that four NLS modes provide a significantly better fit in Figure 4 than do four Fourier modes. The reduction in error, in fact, is close to $60 \%$. The reduction in Figure 5 is significantly less, although still $30 \%$.

\section{Modal reduction}

Often it is desired to describe the dynamics of a specific solution of a partial differential equation using only a finite-dimensional approximation. Such finitedimensional descriptions can be obtained using a variety of methods, all of which are equivalent to a certain order of the approximation, under certain conditions on the perturbed solution and the well-posedness of the partial differential equation.

One approach, useful when the partial differential equation has a variational structure, is variational reduction (see [12] for numerous examples). One introduces an ansatz into the density of the action functional. This ansatz is often a known solution depending on a finite number of parameters, which are allowed to become explicitly time-dependent. This results in a finite-dimensional system of ordinary differential equations for the dynamics of these parameters, obtained by taking variational derivatives with respect to each of the parameters.

Alternatively, again starting from a known solution depending on a finite number of parameters, one may use the method of multiple scales (see [13] for general methodology and [12] for examples in the context of nonlinear waves). After introducing a hierarchy of successively slower time scales, the solution is perturbed by an order $\epsilon$ function ( $\epsilon$ small), while the solution parameters are allowed to depend on the slower times. Substitution of this ansatz in the partial differential equations, separating different orders of $\epsilon$ while accounting for secular terms, results 


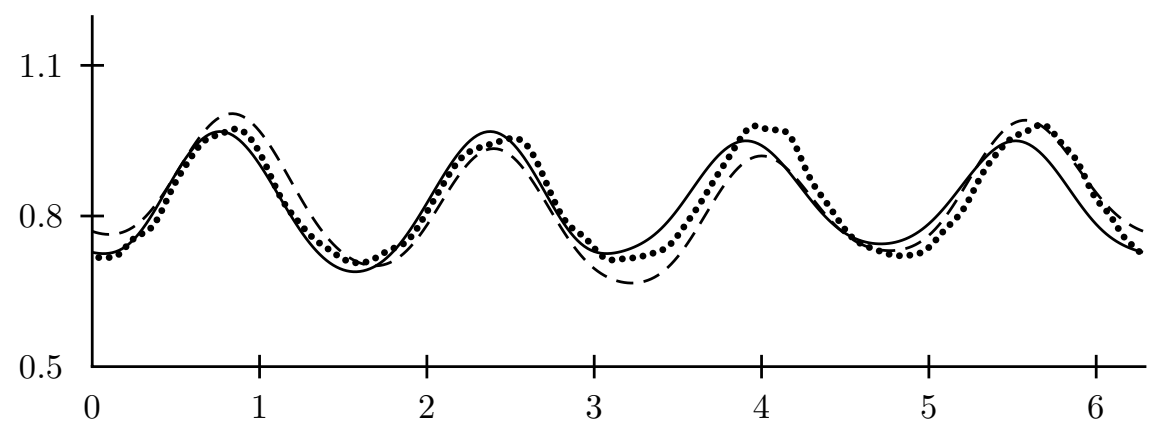

(a)

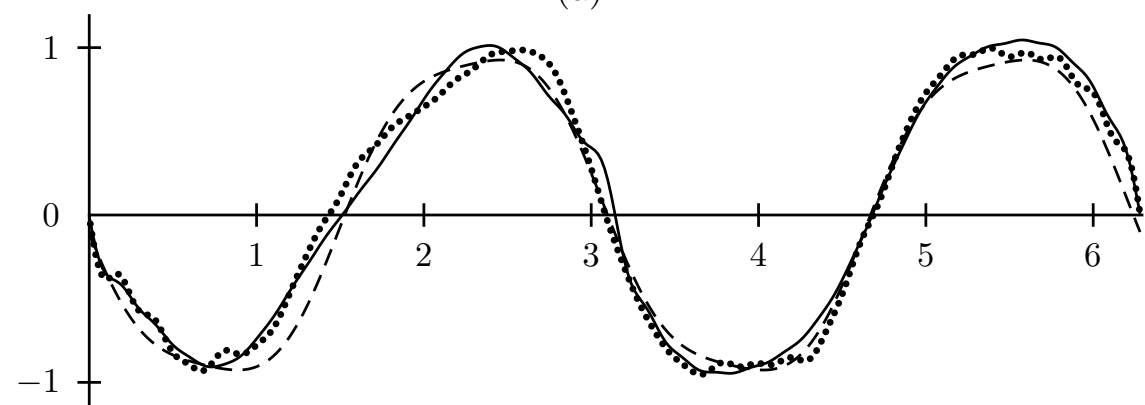

(b)

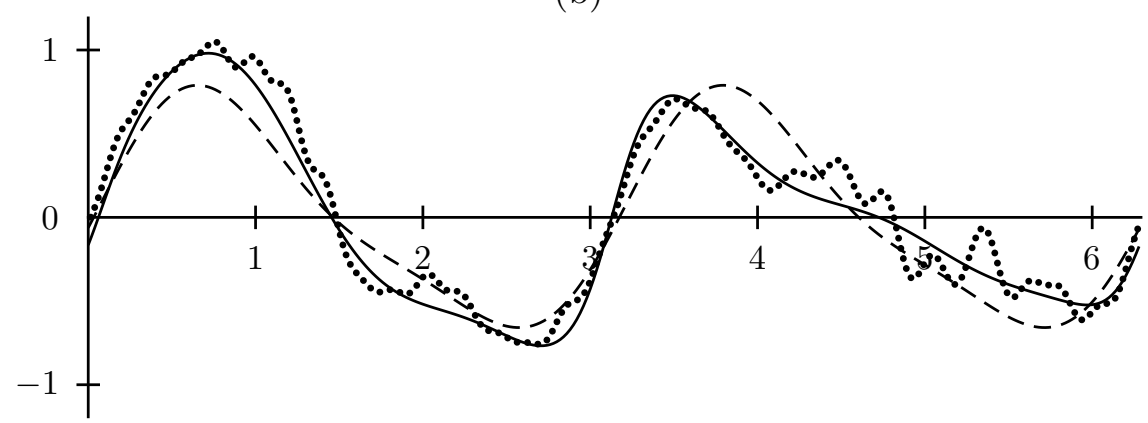

(c)

Figure 3. (a) The absolute value of demodulated time-series data (dotted line), sinusoidal in appearance, from a deep-water wave tank experiment. Using four Fourier modes (dashed), the error in the 2-norm is $0.895 \%$. Using four NLS modes (solid) with $q=0.242$, the error is $.640 \%$. Thus the reduction in error obtained is less than 30\%. (b) Demodulated data (dotted line) from a deep-water wave tank experiment with paddles programmed to produce (using linear theory) the wave profile $.5 \cos (8 \pi x / 182.88) \cos (8 \pi t)$. Using four Fourier modes (dashed), the error in the 2-norm is $2.43 \%$. Using four NLS modes (solid) with $q=0.928$, the error is $1.375 \%$. (c) Demodulated data (dotted line) from a wave tank with paddles programmed to produce the wave profile $.5 \cos (9 \pi x / 182.88) \cos (8 \pi t)$. Using four Fourier modes (dashed), the error in the 2 -norm is $15.05 \%$. Using four NLS modes (solid) with $q=0.454$, the error is $4.31 \%$. For all figures, the horizontal axis displays $t$, scaled to an interval of length $2 \pi$, while the vertical axis shows $\phi$, rescaled to have a magnitude of order one.

in a system of ordinary differential equations for the slow time dependence of the parameters. This method is then truncated at some order.

Both methods rely on the judicious choice of an ansatz solution. We present an alternative to this by using the NLS bases. Our approach has two main advantages. First, there is no guesswork in choosing an ansatz, and no known solution is needed. The method may be used to find solutions of any form. Second, the dimension of the dynamical system obtained may be increased, if so desired, in a straightforward way to achieve arbitrary accuracy, as the solutions are known to converge to those of the PDE as the dimension approaches infinity. Clearly, we do not anticipate this method working well (i.e., provide a good approximation over long time scales using only a low number of modes) for those situations where NLS is not expected to be a good model of the underlying dynamics.

Suppose we are trying to understand the dynamics of the partial differential 


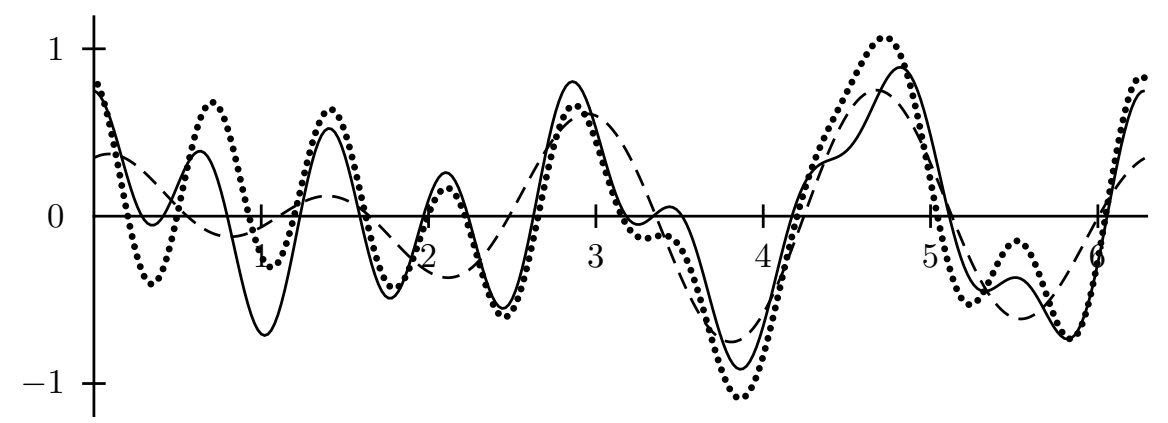

Figure 4. Demodulated time-series data (dotted line) from the large-amplitude wave [18] recorded during Typhoon Krosa, 2007, courtesy of the CWB of Taiwan. The original data was sampled at $2 \mathrm{~Hz}$, with apparent carrier wave at $41 / 512 \mathrm{~Hz}$. Using four Fourier modes (dashed), the error in the 2-norm is $41.98 \%$. Using four NLS modes (solid) with $q=0.669$, the error is $17.3 \%$. The axes are as in Figure 3.

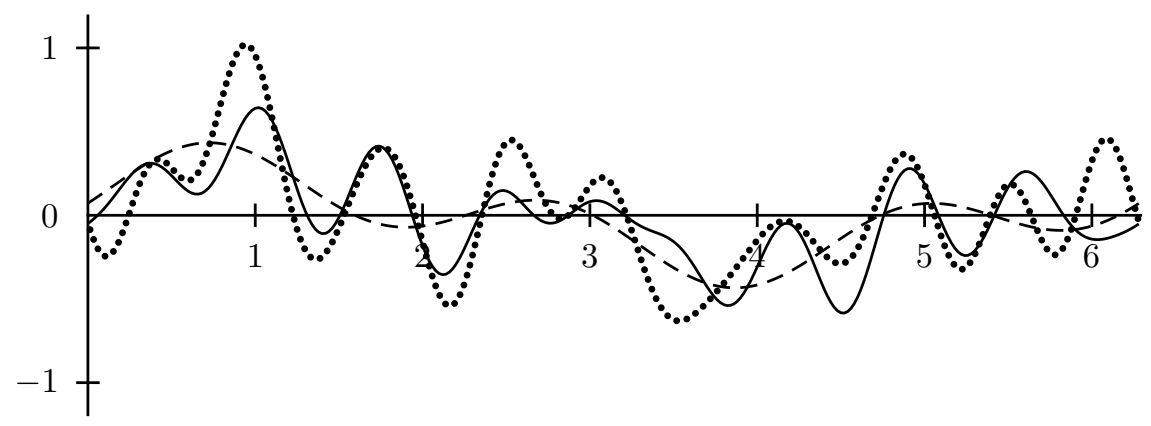

Figure 5. Demodulated time-series data (dotted line) from a Freak wave recorded during Typhoon Krosa, 2007, courtesy of the CWB of Taiwan. The original data was sampled at $2 \mathrm{~Hz}$, with apparent carrier wave at 30/512 Hz. Using four Fourier modes (dashed), the error in the 2-norm is 59.4\%. Using four NLS modes (solid) with $q=0.898$, the error is $41.38 \%$. The axes are as in Figure 3.

equation

$$
u_{t}=N\left(u, u_{x}, u_{x x}, \ldots\right)
$$

with periodic boundary conditions. Here $N\left(u, u_{x}, \ldots\right)$ is a nonlinear (assumed polynomial for simplicity) function of its arguments. We write the solution $u(x, t)$ of (17) as as a series

$$
u(x, t)=\sum_{n=1}^{\infty} c_{n}(t) \phi_{n}(x)
$$

where the $\phi_{n}$ are the NLS modes. Note that the exponential time-dependent factor in (5) is trivially absorbed in the coefficients $c_{n}(t), n \in\{1,2, \ldots\}$. The equation (17) becomes

$$
\sum_{n=1}^{\infty} c_{n}^{\prime}(t) \phi_{n}(x)=N\left(\sum_{n=1}^{\infty} c_{n}(t) \phi_{n}(x), \sum_{n=1}^{\infty} c_{n}(t) \phi_{n}^{\prime}(x), \sum_{n=1}^{\infty} c_{n}(t) \phi_{n}^{\prime \prime}(x), \ldots\right)
$$

Using the orthogonality of the modes $\phi_{n}$, we write this as an infinite-dimensional system of coupled ordinary differential equations. 


$$
c_{\ell}^{\prime}(t)=\frac{1}{L} \int_{0}^{L}\left(\phi_{\ell}^{*}(x) \cdot N\left(\sum_{n=1}^{\infty} c_{n}(t) \phi_{n}^{\prime}(x), \sum_{n=1}^{\infty} c_{n}(t) \phi_{n}^{\prime \prime}(x), \ldots\right)\right) d x .
$$

Truncating the system (19) to limit to a finite number of NLS modes results in a finite-dimensional approximation to the original PDE. Because the coefficients correspond to functions that are stationary with respect to NLS, we may expect this approximation to do well for equations that may be viewed as perturbations of NLS.

A system of ordinary differential equations may also be obtained through a variational approach if (17) originates from an action principle, as described above. Substitution of the ansatz (18) in that action principle results in the same infinitedimensional system for the expansion coefficients. It should be pointed out that if the truncation to a finite number of modes is done at the level of the action principle, before taking the variational derivative, the resulting finite-dimensional systems of ordinary differential equations may not be equivalent.

\section{Example: $Q$ switching in actively mode-locked lasers}

A physical application of a perturbed NLS equation can be found in a mode-locked laser system [23]. Here the dynamics of the electric field envelope $Q$ is given by

$$
i Q_{t}+\left(\frac{1}{2}-i g \tau\right) Q_{x x}+|Q|^{2} Q-i\left(g-M\left(\Gamma-\operatorname{cn}^{2}(\omega T, k)\right)\right) Q=0
$$

The control parameter $g$ is considered a gain, $\tau$ is associated with the fiber amplifier, and $M, \Gamma$, and $\omega$ are associated with the active modulation component. Since this equation has a built in value of the elliptic modulus, no optimization over the elliptic modulus is used to determine the optimal NLS basis. Instead we work with the given value of $k$.

We substitute our series solution in (20) and obtain an $N$-mode truncated system:

$$
\begin{aligned}
c_{\ell}^{\prime}= & -\frac{i}{2} \sum_{m=1}^{N} c_{m} b_{m \ell}+i \sum_{m, n, q=1}^{N} d_{m \ell n q} c_{m} c_{n} c_{q}^{*}+(g-M \Gamma) c_{\ell}+ \\
& M\left(c_{\ell}-\sum_{m=1}^{N} c_{m} a_{\ell m}\right)-g \tau \sum_{m=1}^{N} c_{m} b_{m \ell}
\end{aligned}
$$

where we define

$$
a_{\ell m}=\frac{1}{L} \int_{0}^{L} \phi_{\ell}^{*}(x) \phi_{m}(x) \operatorname{sn}^{2}(x, k) d x, \quad b_{m n}=\frac{1}{L} \int_{0}^{L} \phi_{m}^{\prime}(x) \phi_{n}^{* \prime}(x) d x,
$$

and

$$
d_{m l n s}=\frac{1}{L} \int_{0}^{L} \phi_{l}^{*}(x) \phi_{n}(x) \phi_{s}^{*}(x) \phi_{m}(x) d x
$$




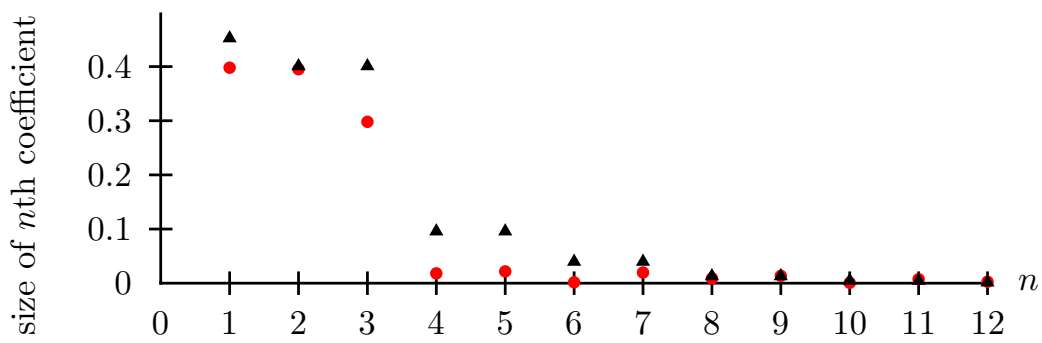

Figure 6. Starting with all energy equally split among the first three modes $\left(c_{1}=c_{2}=c_{3}=0.4\right.$, with $c_{j}=0, j>3$ ), we evolve the system to $t=0.15$. The dots show the absolute value of each coefficient of the NLS eigenfunctions (circles) and Fourier modes (triangles).

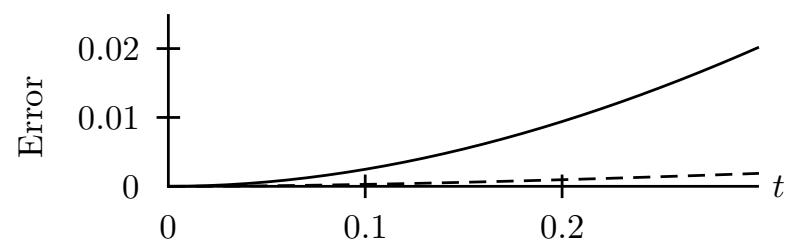

Figure 7. The error (the fraction of the 2-norm that is lost) by considering only the first three modes, as a function of time, for the numerical simulation represented in Figure 6. The solid line represents taking the first three Fourier modes, while the dashed line, with a lower error, represents the use of three NLS eigenfunctions.

If this approach is to be effective, the contribution from the neglected coefficients must not become large on short time scales. To test this, we compare with the performance of an ODE system based on the coefficients of Fourier modes. In Figure 6, we see an example where the energy in an initial condition does not spread as quickly to other modes in our system as in the Fourier system. This result has been found to be true for many other initial conditions as well. In Figure 7, the time evolution of the error as a function of time is displayed, showing the faster increase in error when using Fourier modes, compared to using NLS modes. The collection of these results indicates that the NLS basis may be a natural, or at least convenient, basis for solutions of (20).

\section{Example: the dynamics of NLS}

As a final example, we apply the NLS basis decomposition to NLS itself. This example is meant as a mere illustration of the methods, since NLS is very efficiently solved using other means, being an integrable equation.

In the case of NLS we have $N\left(u, u_{x}, u_{x x}\right)=i u_{x x}+2 i \sigma|u|^{2} u$, which yields

$$
c_{\ell}^{\prime}(t)=-i \sum_{n=1}^{\infty} b_{l n}^{*} c_{n}(t)+2 i \sigma \sum_{n, m, s=1}^{\infty} d_{m n l s}^{*} c_{m}^{*}(t) c_{n}(t) c_{s}(t) .
$$

This result can also be obtained by equating the variation of the Lagrangian of NLS with respect to $c_{\ell}$ to 0 .

If we truncate the series in (21) to a finite number of terms, we obtain a finitedimensional ODE system that approximates the dynamics of NLS. All that has been used about the functional basis in the derivation of (21) is completeness and orthonormality. Hence the formula holds for a Fourier basis as well, though the coefficients $b_{m n}$ and $d_{m l n s}$ have simpler analytic expressions. For Fourier coefficients, $b_{m n}=0$ for $m \neq n$, and $d_{m l n s}=0$ unless $m+n-l-s=0$. This indicates that energy may have less of a tendency to spread among modes than in the case of 
NLS modes, where more of these coefficients are nonzero.

A typical numerical result is shown in Figure 8. Note that this plot really depicts two separate initial conditions. But starting with energy in the first three Fourier modes, the energy does not spread to other modes as much as when starting with energy in the first three NLS modes.

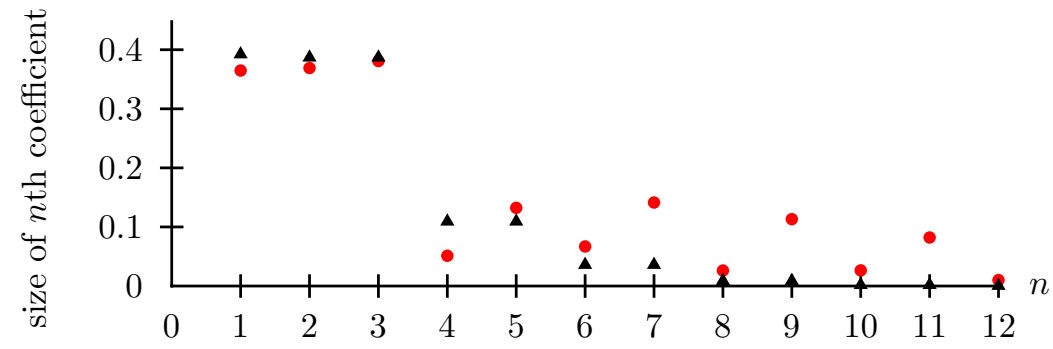

Figure 8. Time evolution is run with energy initially in the first three modes $\left(c_{1}=c_{2}=c_{3}=0.4\right.$, with $c_{j}=0, j>3$; eigenfunctions or Fourier modes). The circles show eigenfunction coefficients at $t=0.1$; the triangles show results with Fourier modes.

As an added remark, it should be stated that the system (21) is an example of a non-autonomous integrable lattice system. Clearly, it is integrable, due to its immediate relationship with the NLS equation. However, it is of a different type than any of the typical integrable lattice equations such as the Toda lattice and the Ablowitz-Ladik lattice [1]. Of course, this remark is in order for (17), no matter which basis is used to reduce the equation.

\section{Conclusion}

We have introduced a one-parameter family of bases for the square integrable functions on a finite interval. Different bases are characterized by different values of the elliptic modulus $k \in[0,1)$. For $k=0$, the classical Fourier basis is recovered. The optimal basis for a given application is found by optimizing an error functional over $k$. The elements of the new bases are stationary solutions of the NLS equation. Because of this, it is anticipated that the new bases might be especially useful when investigating the dynamics of phenomena that are well described by perturbations of the NLS equation. This is investigated using different examples from deep-water waves (using data analysis) and nonlinear optics (modal reduction).

\section{Acknowledgements}

The authors wish to thank Megan Catalano, Diane Henderson and Paul C. Liu, for providing us with the data used in Section 4.2. Furthermore, the authors acknowledge support from the National Science Foundation through grant NSF-DMS0604546 (BD) and NSF-DMS-VIGRE- 0354131 (DOL). Any opinions, findings, and conclusions or recommendations expressed in this material are those of the authors and do not necessarily reflect the views of the funding sources.

\section{References}

[1] M. J. Ablowitz and P. A. Clarkson, Solitons, nonlinear evolution equations and inverse scattering, Cambridge University Press, Cambridge, 1991.

[2] L. D. Carr, C. W. Clark, and W. P. Reinhardt, Stationary solutions of the one-dimensional nonlinear Schrödinger equation: I. Case of repulsive nonlinearity, Phys. Rev. A 62 (2000), pp. 063610 
[3] L. D. Carr, C. W. Clark, and W. P. Reinhardt, Stationary solutions of the one-dimensional nonlinear Schrödinger equation: II. Case of attractive nonlinearity, Phys. Rev. A 62 (2000), pp. 063611

[4] J. D. Carter and B. Deconinck, Instabilities of one-dimensional trivial-phase solutions of the twodimensional cubic nonlinear Schrödinger equation, Physica D 214 (2006), pp. 42-54.

[5] E. W. Cheney, Introduction to approximation theory, McGraw-Hill Book Co., New York, NY, 1966.

[6] R. Conte and M. Musette, The Painlevé handbook, Springer, Dordrecht, 2008.

[7] B. Deconinck and J. N. Kutz, Computing spectra of linear operators using the Floquet-Fourier-Hill method, J. Comput. Phys. 219 (2006) pp. 296-321.

[8] J. Hammack, D. Henderson, and H. Segur, Progressive waves with persistent, two-dimensional surface patterns in deep water, J. Fluid Mech. 532 (2005), pp. 1-51.

[9] T. Gallay and M. Haragus, Orbital stability of periodic waves for the nonlinear Schrödinger equation, J. Dyn. Diff. Eqns. 19 (2007), pp. 825-865.

[10] T. Galley and M. Haragus, Stability of small periodic waves for the nonlinear Schrödinger equation, J. Diff. Eqns. 234 (2007), pp. 544-581.

11] E. L. Ince, Ordinary differential equations. Dover Publications, New York, NY, 1956.

[12] E. Infeld and G. Rowlands, Nonlinear waves, solitons and chaos, Cambridge University Press, Cambridge, 2000.

[13] J. Kevorkian and J. D. Cole, Multiple Scale and Singular perturbation methods, Springer-Verlag, New York, NY, 1996.

[14] M. Kirby, Geometric data analysis, An empirical approach to dimensionality reduction and the study of patterns, Wiley-Interscience, New York, NY, 2001.

[15] I. M. Krichever, Spectral theory of two-dimensional periodic operators and its applications, Russian Math. Surveys 44 (1989), pp. 145-225.

[16] D. F. Lawden, Elliptic functions and applications, Springer-Verlag, New York, NY, 1989.

[17] B. M. Levitan and I. S. Sargsjan, Introduction to spectral theory, American Mathematical Society, Providence, RI, 1975.

[18] P. C. Liu, H.S. Chen, D.-J. Doong, C.C. Kao, and Y.-J.G. Hsu. Monstrous ocean waves during Typhoon Krosa, Annales Geophysicae 26 (2008) pp. 1327-1329.

[19] E. N. Lorenz, Deterministic nonperiodic flow, J. Atmos. Sci. 20 (1963), pp. 130-141.

[20] W. Magnus and S. Wingler, Hill's equation, Dover Publications, New York, NY, 1979.

[21] P. J. Olver, Applications of Lie groups to differential equations. Springer-Verlag, New York, NY, 1993.

[22] M. Onorato, A. R. Osborne, M. Serio, and L. Cavaleri, Modulational instability and non-Gaussian statistics in experimental random water-wave trains, Phys. of Fluids 17 (2005), pp. 078101.

[23] J. L. Proctor and J. N. Kutz, Theory of q switching in actively mode-locked lasers, J. Opt. Soc. Am. B 23 (2006), pp. 652-662.

[24] H. Segur, D. Henderson, J. D. Carter, J. Hammack, C.-M. Li, D. Pheiff, and K. Socha, Stabilizing the Benjamin-Feir instability, J. Fluid Mech. 539 (2005), pp. 229-271.

[25] E. T. Whittaker and G. N. Watson, A course of modern analysis, Cambridge University Press, Cambridge, 1927. 